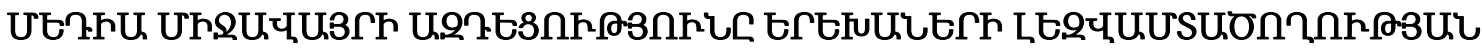

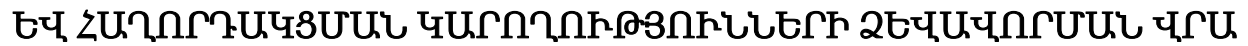

UUนกคЧзUน U. U.

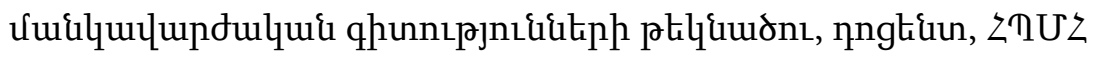

૧UしKELBUし 9. L.

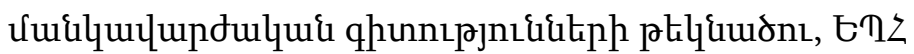

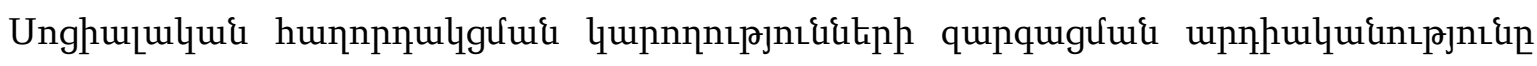

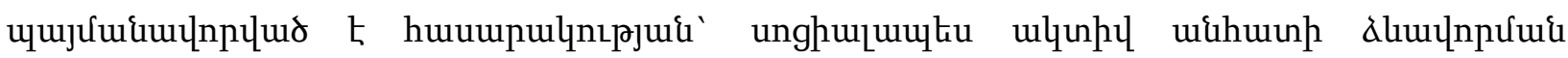

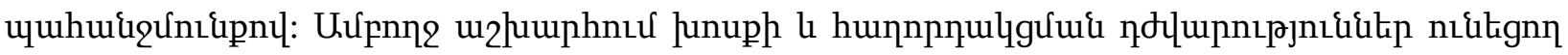

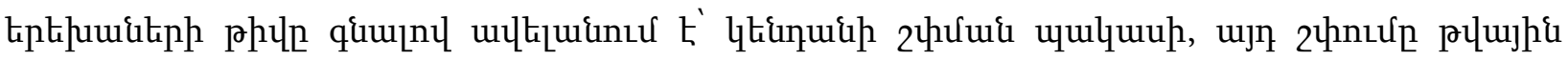

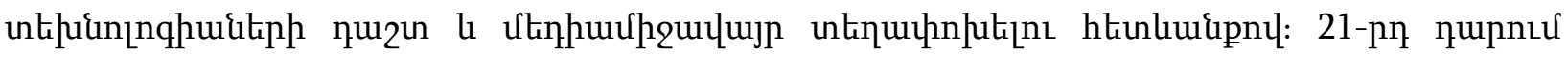

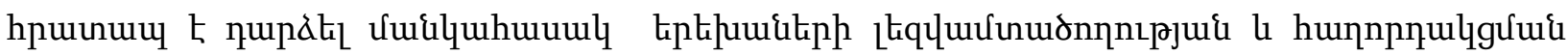

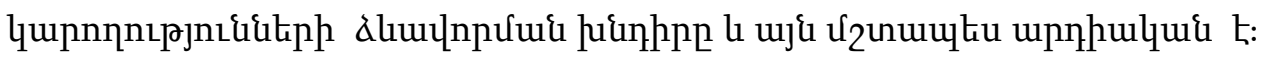

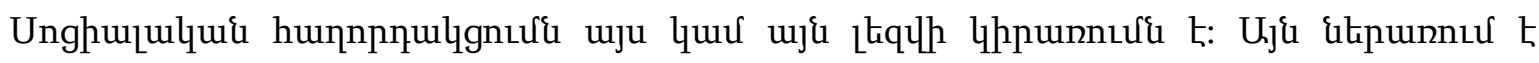

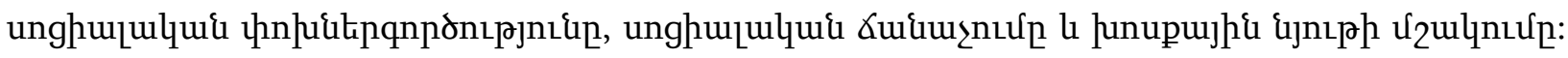

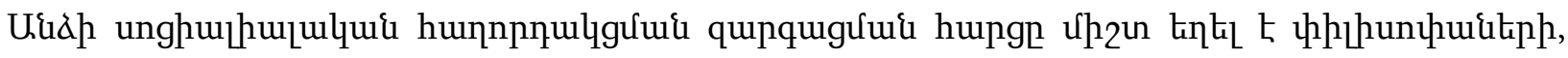

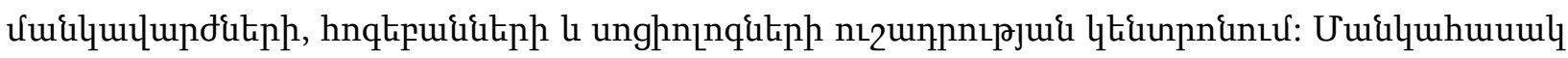

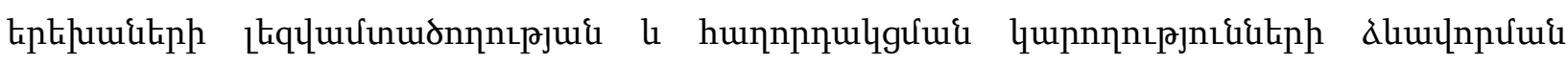

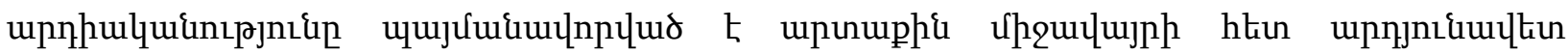

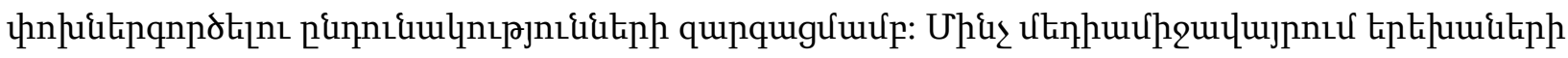

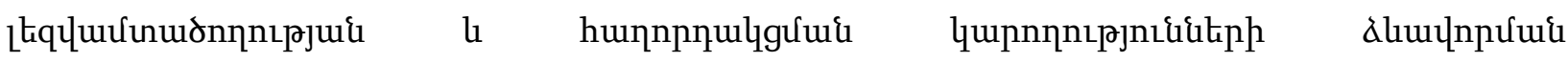

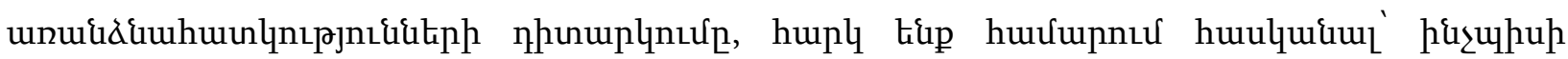

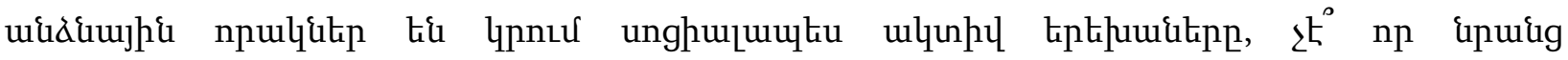

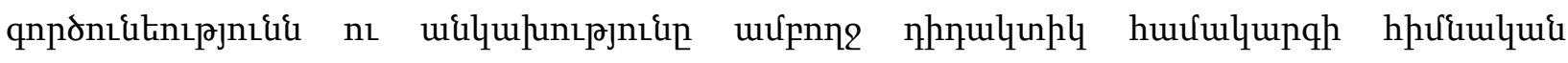

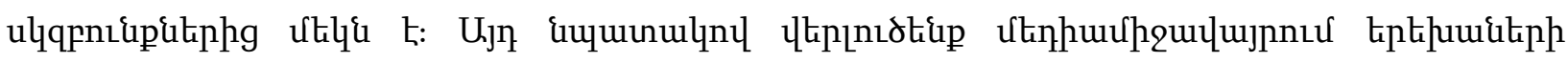

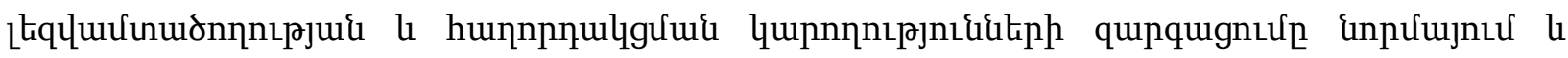

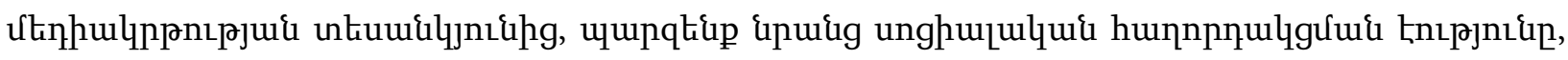

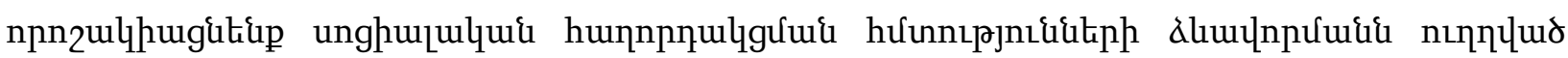

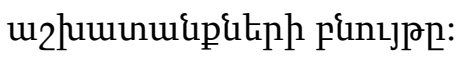

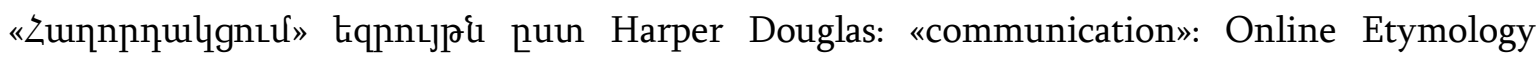

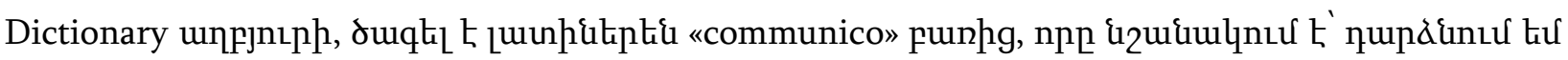




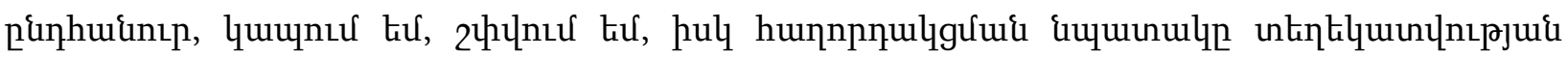

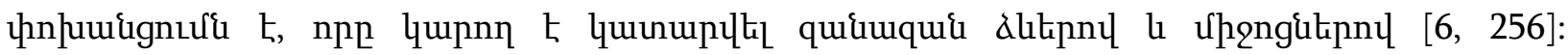

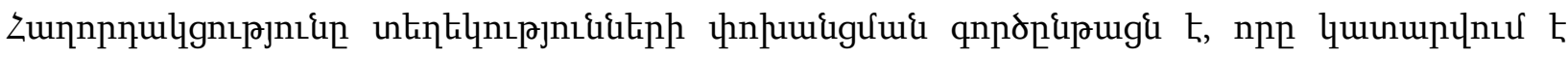

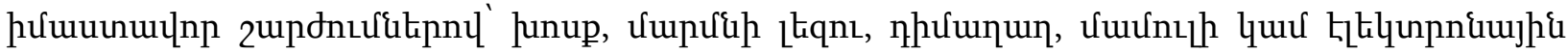

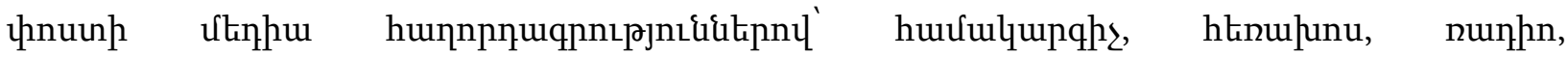

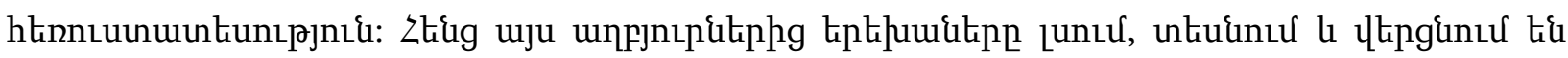

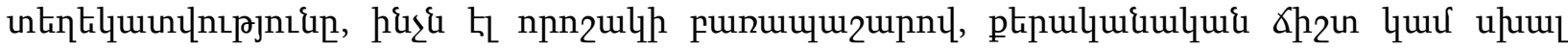

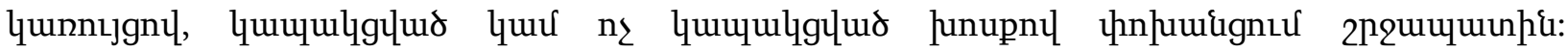

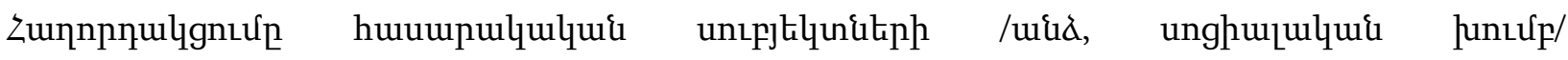

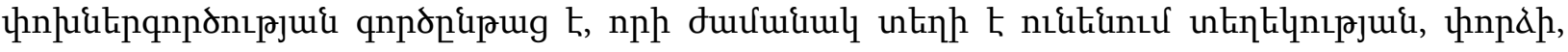

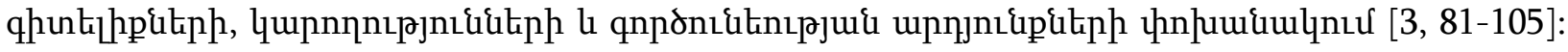

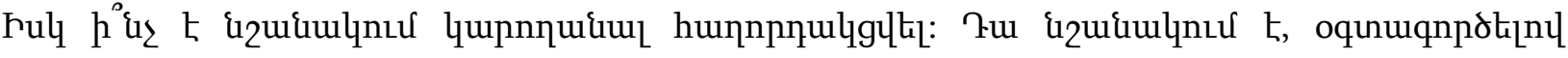

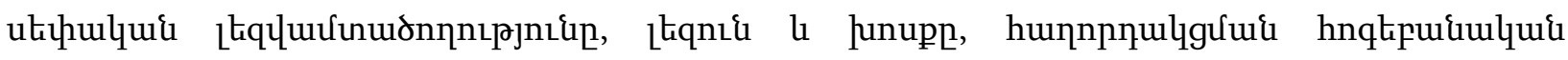

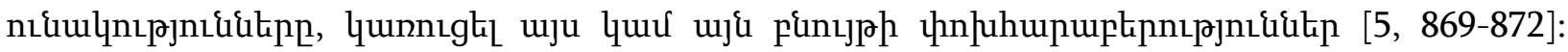

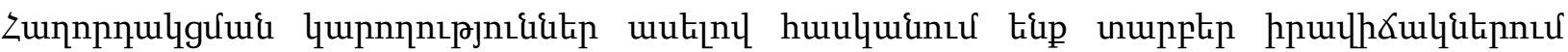

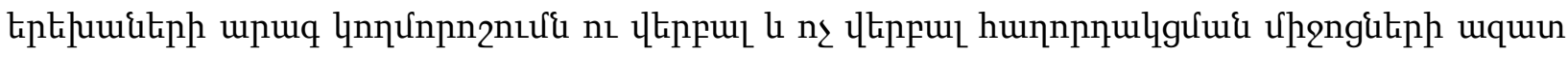

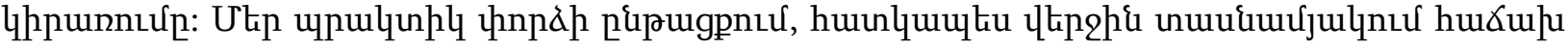

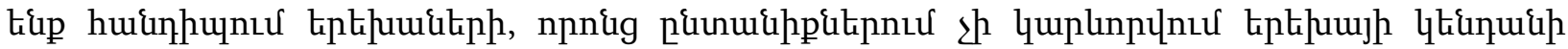

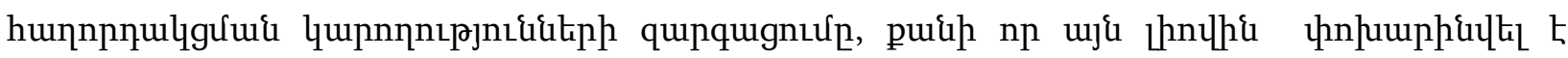

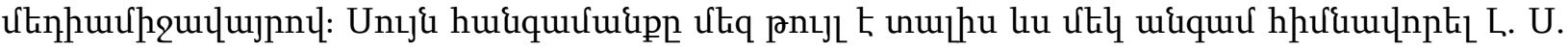

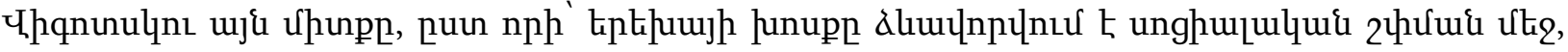

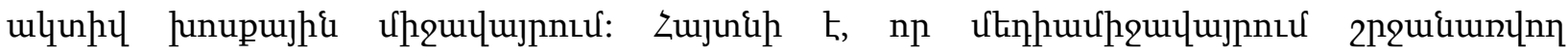

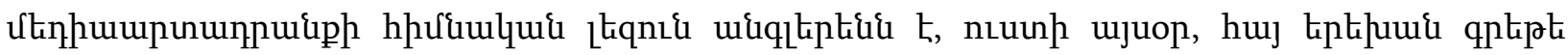

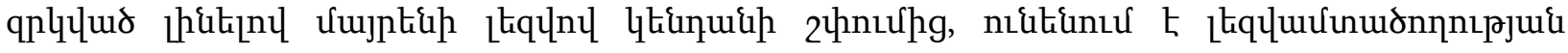

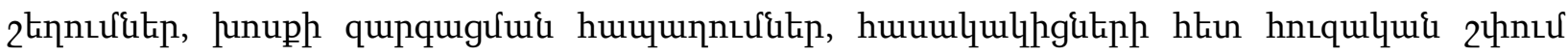

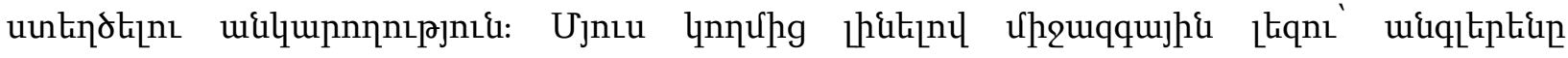

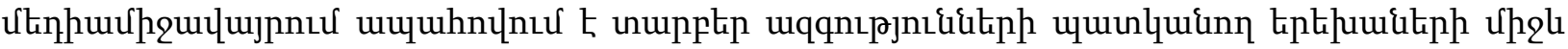

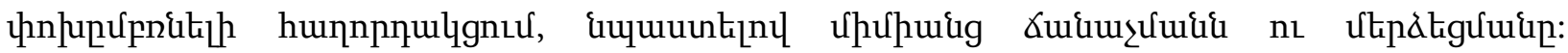

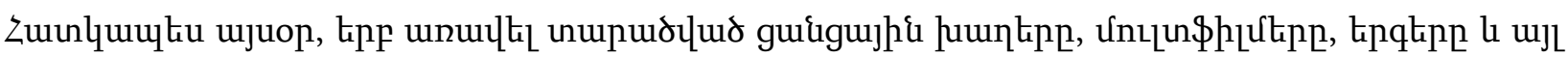

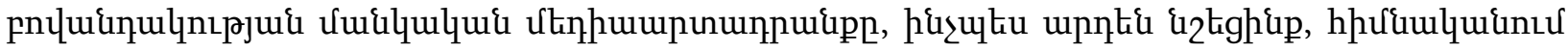

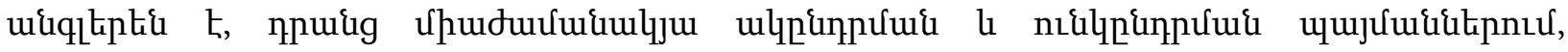

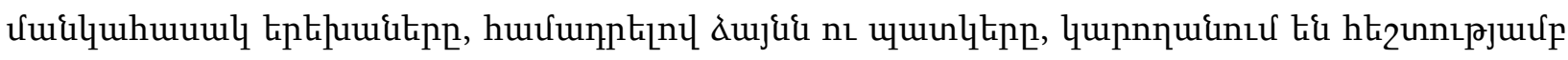

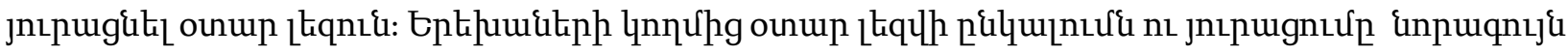




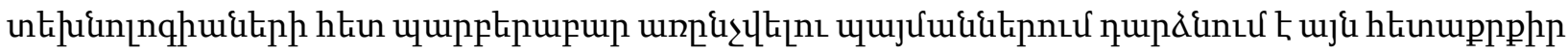

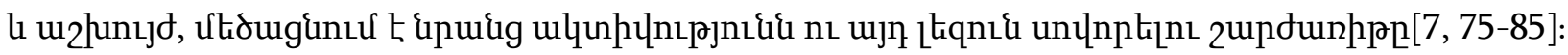

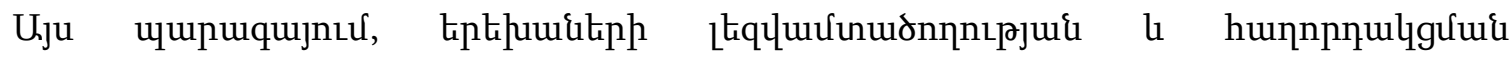

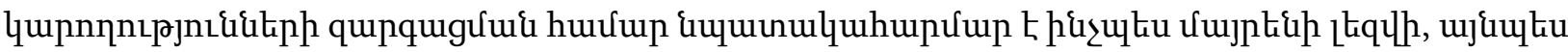

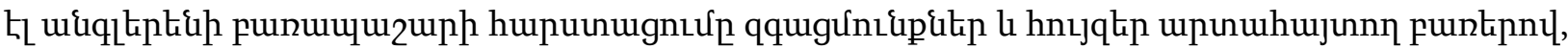

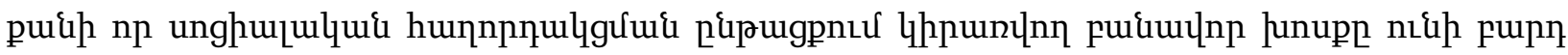

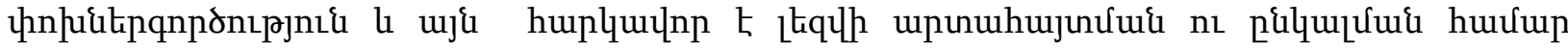

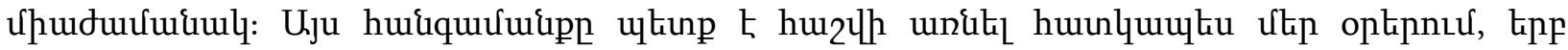

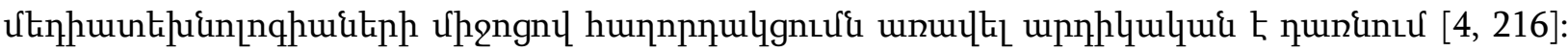

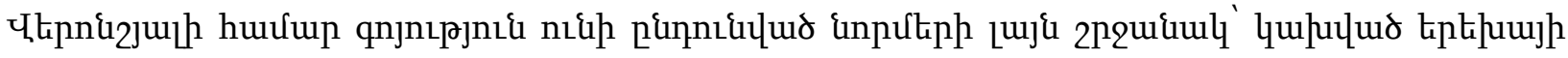

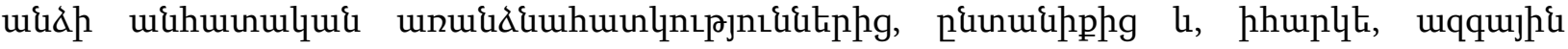
ưuulquiliŁhnıpjnı\{hg:

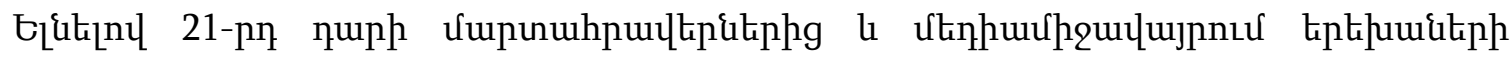

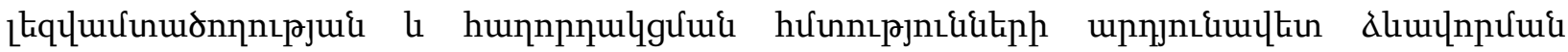

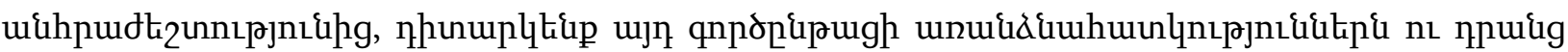

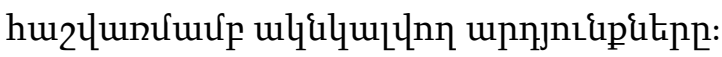

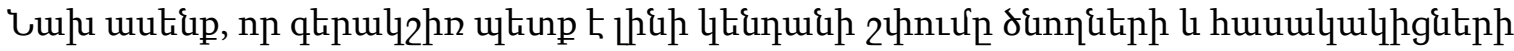

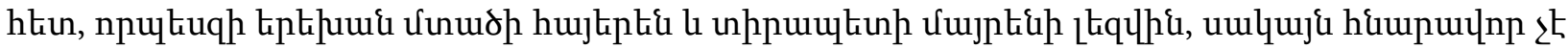

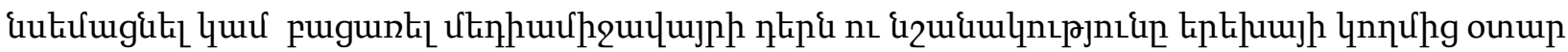

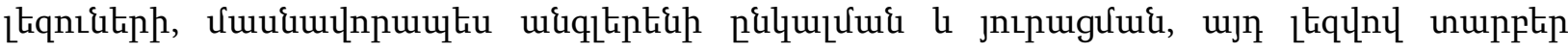

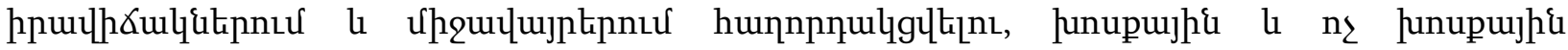

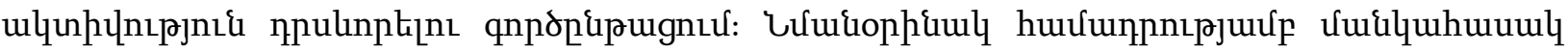

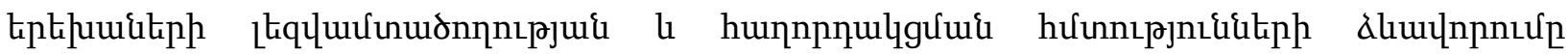
hqumulnpntpjnı\&

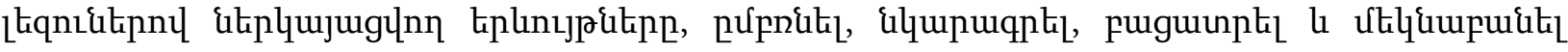

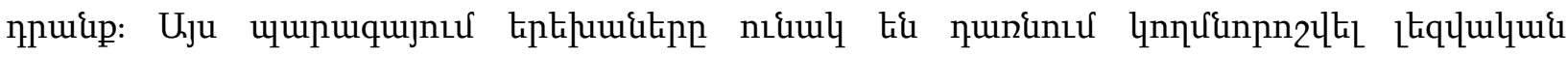

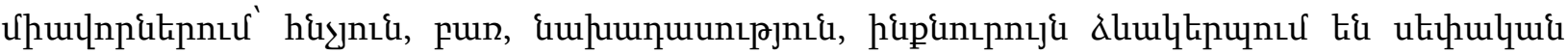

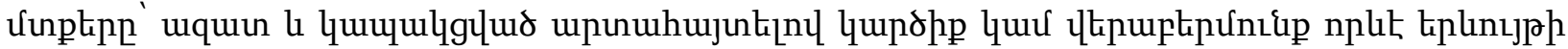

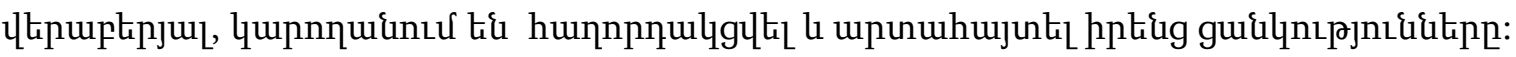

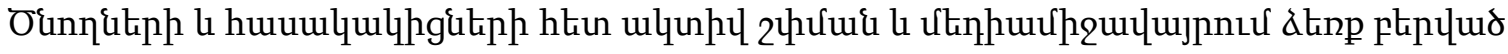

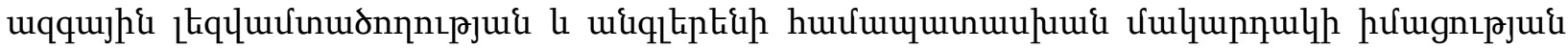

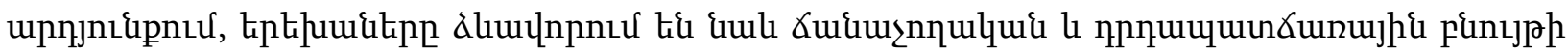

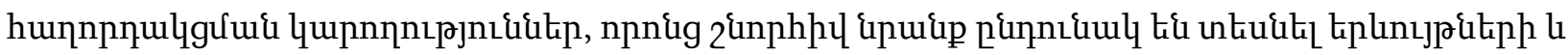




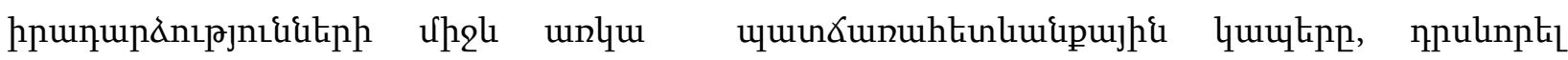

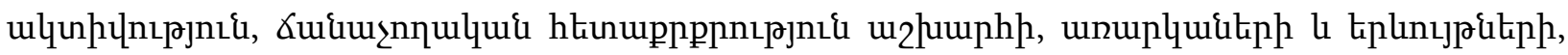

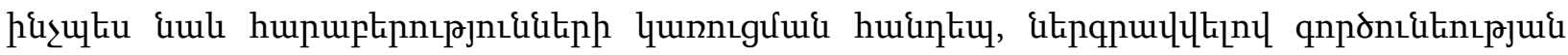

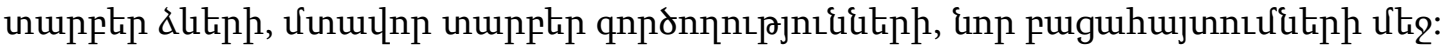

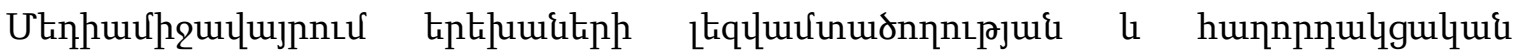

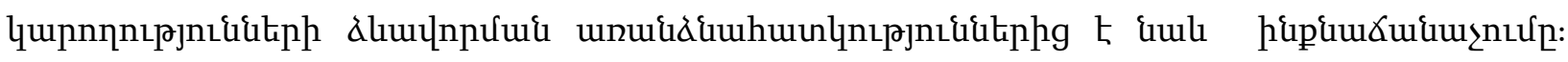

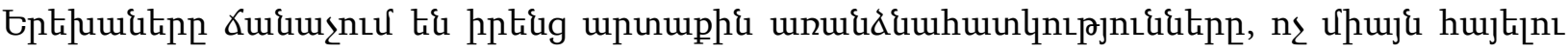

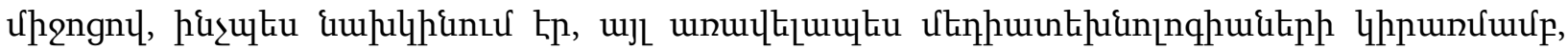

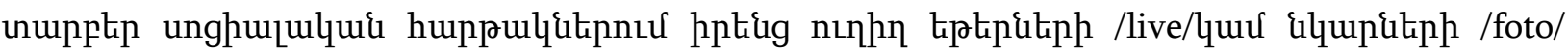

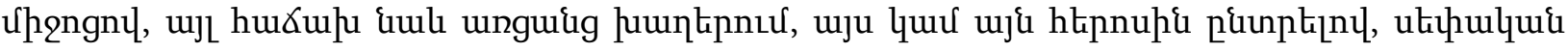

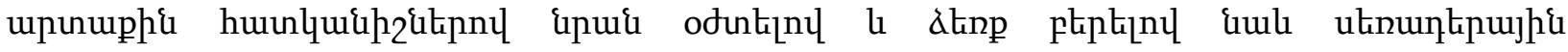

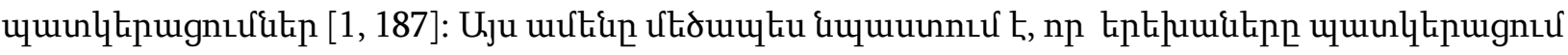

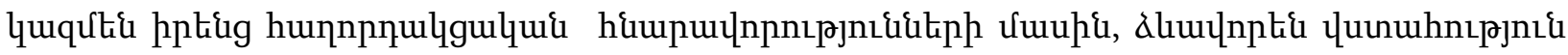

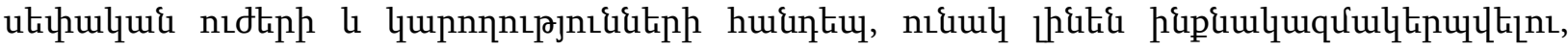

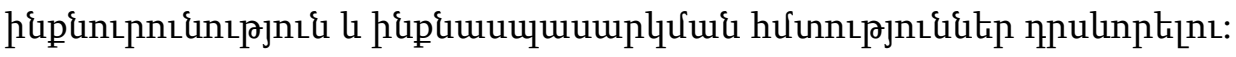

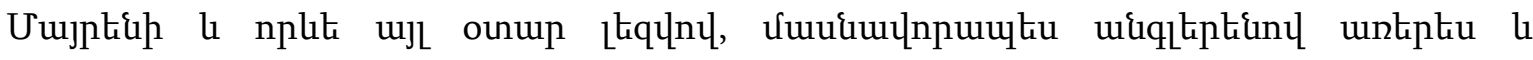

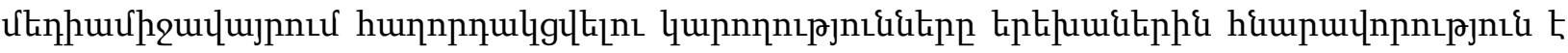

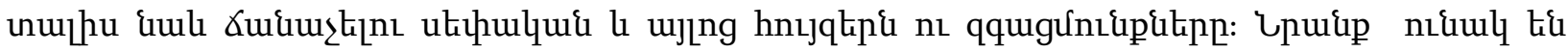

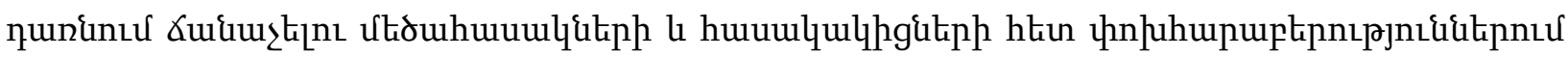

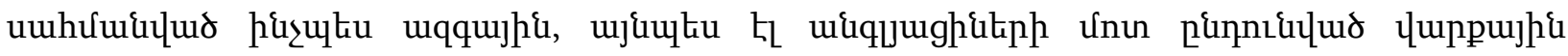

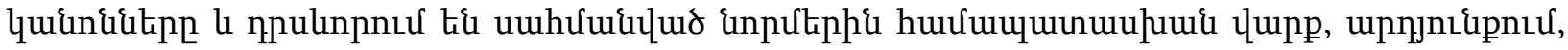

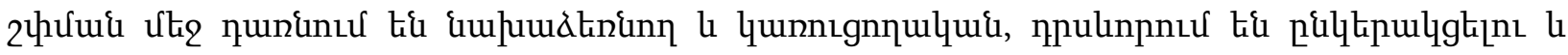

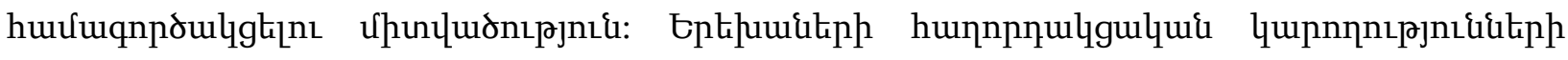

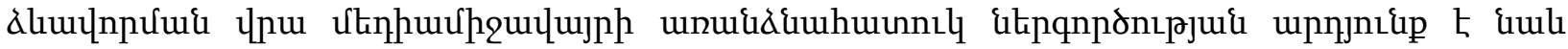

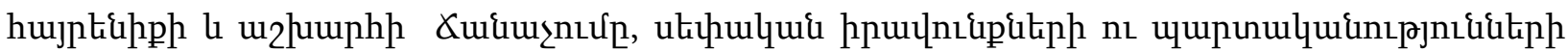

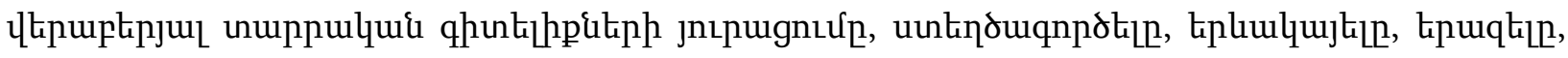

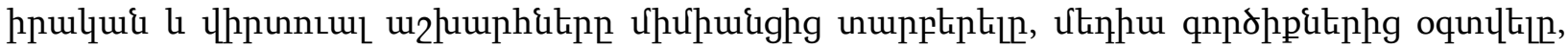

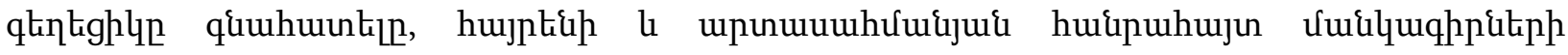

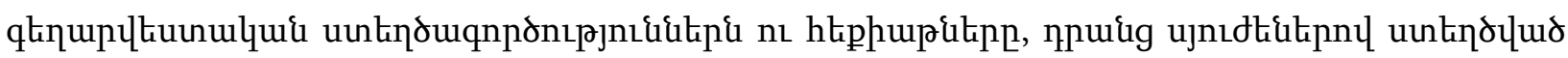

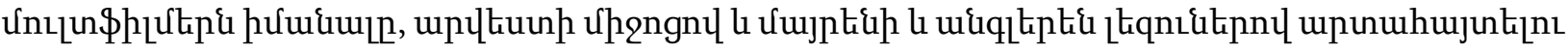

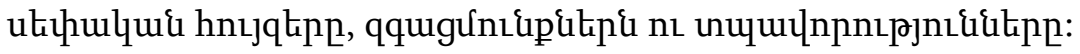

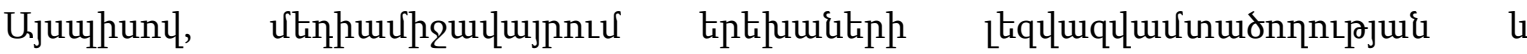

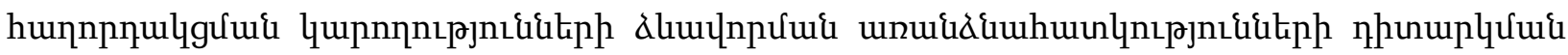




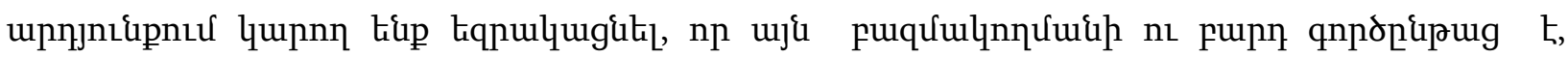

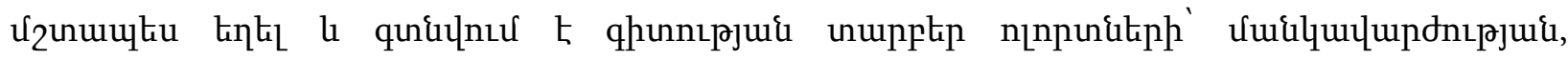

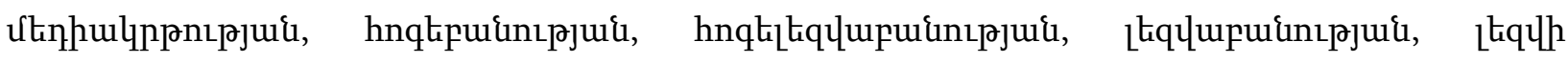

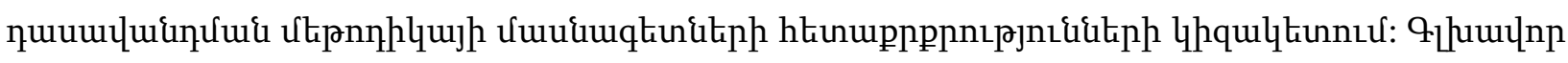

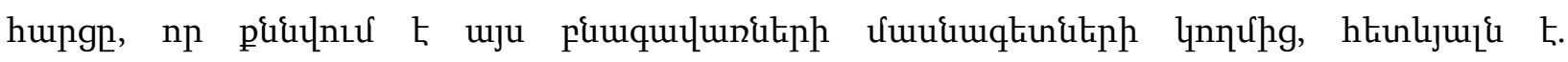

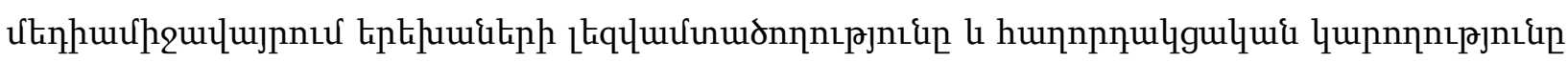

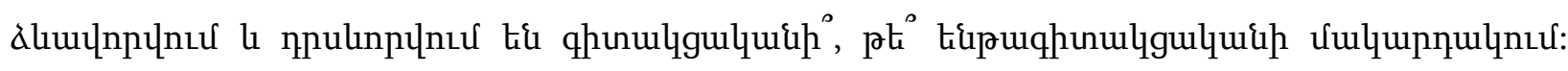

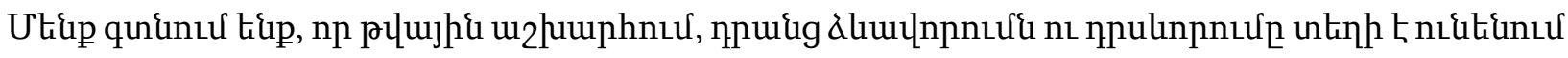

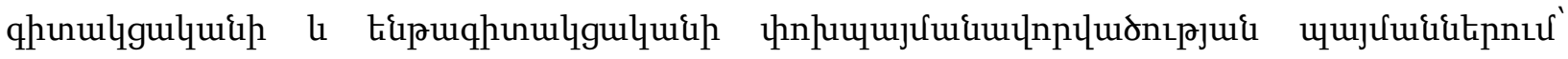

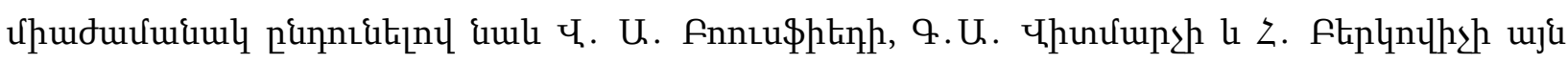

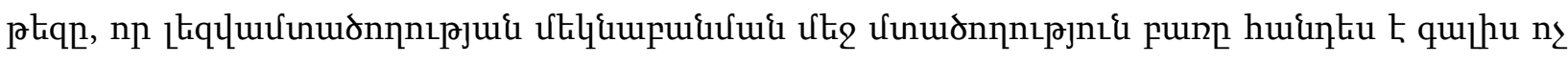

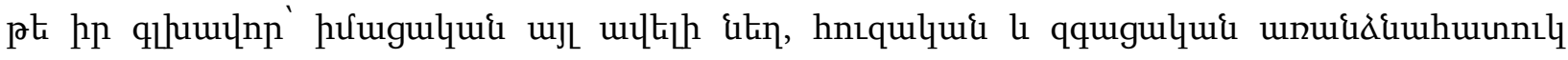

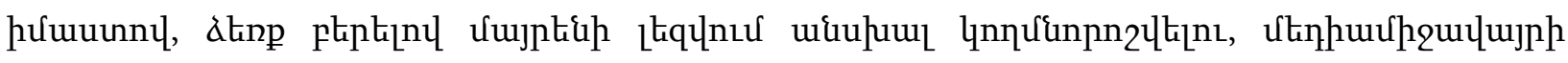

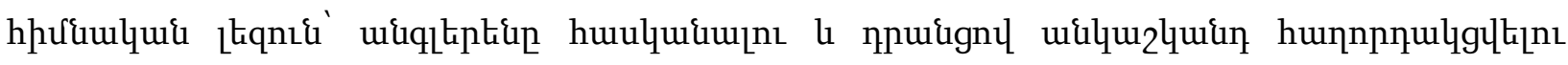

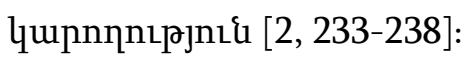

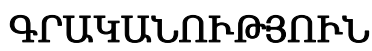

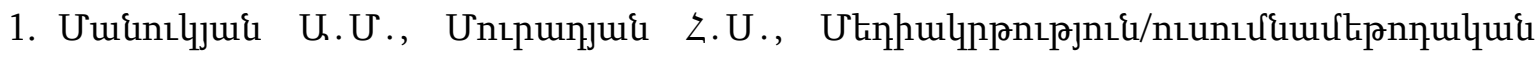

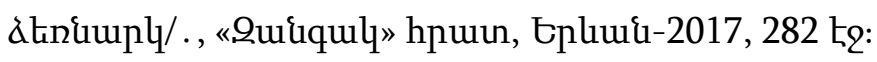

2. Bousfield W.A., Whitmarch G.A., Berkowitz H., Partial responses identities in associative clustering, J. of general psychology, v. 63, 1960, pp. 233-238.

3. Campbell L., The History of Linguistics, The Handbook of Linguistics, «Mark Aronoff. Blackwell», 2001, pp. $81-105$.

4. Cofer Sh. N., Associative commonality and rated similarity of certain words from Hagen's list, Psychological reports, v. 3, 1957, pp. 603 - 606. Deese J., The structure of associations in language and thought. Baltimore, «J.Hopkins press», 1966, pp. 216.

5. Dolinsky V.A., Quantitative Model for Word Association Data, SPECOM'2007, the XIIth Inter. Conf. —Speech and Computerl, Proc., V. II. M., 2007, pp. 858-866. Enard W., Przeworski M., Fisher .S, Lai C, Wiebe V ., Kitano T., Monaco A., Pääbo S., Molecular evolution of FOXP2, a gene involved in speech and language, Nature, 418(6900), 2002, pp. $869-872$

6. Gardner H., Truth, beauty, and goodness reframed: Educating for the virtues in the 21st century, New York: Basic Books, 2011, 256 p.

7. Howes D., On the relation between the probability of a word as an association and in general 
verbal usage, J. of abnormal and social psychology, v. 54, 1957, pp. 75-85.

\section{FEATURES OF CHILDREN'S LANGUAGE AND COMMUNICATION CAPACITIES IN THE MEDIA ENVIRONMENT}

The article presents the results of observing the peculiarities of children's language thinking and communication skills development in the media environment, based on which we conclude that it is a multifaceted and complex process that has always existed in various fields of science: pedagogy, media education, psychology, linguistics, linguistics, linguistics, linguistics. in the focus of specialists' interests. There is a belief that the main issue being examined by experts in these fields is that in the digital world, the development and manifestation of children's language thinking and communication skills takes place in the conditions of conscious and subconscious mutual agreement.

Keywords: media education, children, language thinking, social communication, media technologies, body language, psycholinguistics, family. 